\title{
Conceptual Design of Service-Based Geospatial Information System
}

\author{
Amarsaikhan Damdinsuren ${ }^{1, *}$, Odontuya Gendaram ${ }^{2}$, Enkhjargal Damdinsuren ${ }^{1}$, \\ Bolor Gendaram ${ }^{3}$, Tsogzol Gurjav ${ }^{1}$, Bat-Erdene Tsedev ${ }^{4}$ \\ ${ }^{I}$ Institute of Geography and Geoecology, Mongolian Academy of Sciences, Ulaanbaatar, Mongolia \\ ${ }^{2}$ Mongolian University of Pharmaceutical Sciences, Ulaanbaatar, Mongolia \\ ${ }^{3}$ Intelsom Impex LLC, Ulaanbaatar, Mongolia \\ ${ }^{4}$ Mongolian National University of Education, Ulaanbaatar, Mongolia \\ *Corresponding author.Email: amarsaikhan@mas.ac.mn
}

\begin{abstract}
At the present time, geospatial information systems are widely used for diverse spatial related decision-making processes. The power of these systems comes from the ability to relate different information in a spatial context, placing the information at a given reference system, and reaching conclusions about the relationships. Different types of such systems are designed at national, regional and local level considering the objectives of the target projects or purposes of the implementing agencies. Database structures of the systems can be extremely complex or very simple depending upon the functions of the organizations and user requirements. The aim of this study is to describe a conceptual framework and system design related to the service-based geospatial information system in Mongolia.
\end{abstract}

Keywords: Conceptual design, database function, geospatial information system

\section{INTRODUCTION}

Generally, the geoinformation is a pre-requisite for any spatial planning and management, and a key consideration for many decision-making processes. It is usually generated from different internal and external sources and includes several important aspects such as spatial extent, attributes, relationships, and quality [1].

The geographic dimension is of great importance and it increases the need of managing spatial information. The applications and use of such information have great social and economic relevance [2].

In recent years, consumer demand has increased tremendously for spatial information and tools like GIS to manipulate and display geographical information. The explosion of consumer demand for spatial information has brought its management systems into the daily lives of millions of people around the world. In addition, it is worth to mention that many government, federal and state organizations as well as private entities utilize different types of GIS systems depending on the type of the business functions [3].

In Mongolia, there are different needs to develop online service oriented information systems used for different decision-making processes. In the country's context, the system can be implemented as a webbased dynamic software solution to enable the effective information sharing among stakeholders and support national sector development operations [4].

Data sharing is important to avoid data redundancy and work duplication, thus saving time and labor cost [5]. Moreover, it can optimize the use of organization's limited resources which is a very important feature for most developing countries.

The aim of this study is to describe conceptual designs of an online service-based geospatial information system and its database structures in Mongolia. 


\section{CONCEPTUAL DESIGN OF DATABASE}

Database is a collection of data stored in a way that makes data easily accessible. Database design is the process of producing a detailed data model of database. In most cases, design of efficient and wellstructured database includes such steps as requirements analysis, find the information required, and divide the information into tables, specifying primary keys and analyzing relationships, and normalizing to standardize the tables [6].

Understanding the purpose of the database is a very important step. To gather information before creating the database, the design experts should conduct extensive research study. Initially, we had to identify the purpose of database and assumed that there is a need to develop the database to support information sharing and economic sector marketing. It should contain the product and service information of different enterprises from rural Mongolia. By reviewing the existing documents, discussing with different experts, and interviewing necessary people, we have identified the required information and data sets. Usually, the database modelling follows two stages, namely conceptual model and physical model.

Conceptual model describes a conceptual framework for the abstraction, simplification and classification of the phenomena and their relationships as viewed by the user community of the database. At this stage, a logical structure which specifies the logical data content of the database should be defined. For proper conceptual database design one should clearly define data sets and differentiate all possible entities and their attributes. At this stage, classification and grouping of classes of objects, the reduction of redundancies or duplications have to be thoroughly investigated.

The database should consist of two types of datasets: spatial and attribute. For the representation of spatial datasets a layer-based approach can be used. For proper database design and implementation different issues related to the themes (eg, how many, symbols and annotation, relationships and identifiers) and their attributes (IDs, relationships) should be replicated. For the attribute database design, traditionally data flow diagrams, flow charts and entity relationship diagram (ERD) could be used. Of these, the ERD still has wide applications [7].

In the case of spatial data, the zone (polygon), aimag (point), and soum (point) areas could be defined as spatial entities (Figure 1). Then, for all entities, the attributes could be defined. Moreover, they should be uniquely identified by their ID numbers. In the case of attribute database design, the ERD might be used.

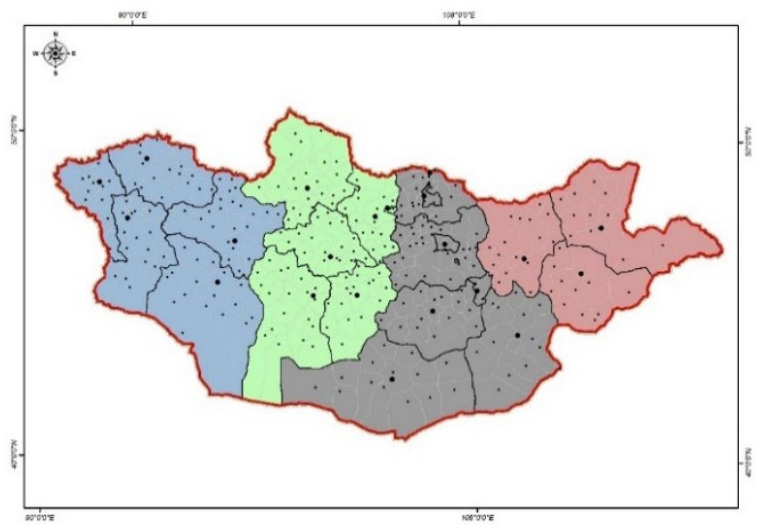

Figure 1. Map of zone, aimag, and soum areas of Mongolia

It can be illustrated as shown in Figure 2. As seen from the Figure 2, we identified the highest-level relationships among the entities. Now we have to carry out the following activities:

1. Specify primary keys for all entities

2. Find the relationships between the entities

3. Find all attributes for each entity

4. Specify foreign keys for all entities

5. Conduct normalization, if necessary.

Based on the ERD and conducted requirements analysis, we can identify the following entities and attributes:

1. Country (ID, name, zoneID)

2. Zone (ID, name, aimagID)

3. Aimag (ID, name, ..., zoneID)

4. Soum (ID, name, zipcode,..., aimagID)

5. Bag (ID, name, code, zipcode,..., aimag_ID, soumID)

6. Company (ID, name, sector, activity,...,aimagID, soumID, bagID)

7. Product (ID, name, stock, assortment, item,..., companyID)

8. Service (ID, name, assortment, item,.., companyID).

As seen from the above, the entities are uniquely identified by their IDs and the relationships between the entities are one to many $(1: \mathrm{m})$. The IDs of the entities of lower hierarchy could be used as foreign keys or posted IDs.

In order to be efficiently implemented within database, the above entities should be converted to (class) tables, and then normalized. Normalization is a technique to organize tables in a manner that 
reduces redundancy and dependency of data. That is normalization is used to eliminate redundant data and improve data integrity [8]. It divides larger tables into smaller tables and links them using relationships. The most commonly used normal forms are the following:

- $\quad$ First normal form (1NF)

- $\quad$ Second normal form (2NF)

- $\quad$ Third normal form (3NF)

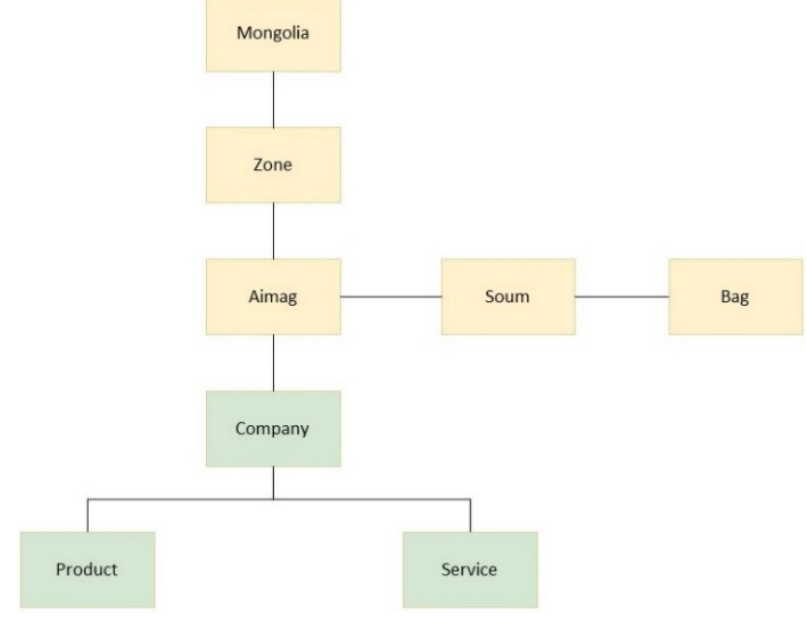

Figure 2. Conceptual model of the database

Within the framework of the present study, we applied the normalization up to a second normal form and the result is shown in Figure 3 (details are represented in a UML diagram shown in a physical model).

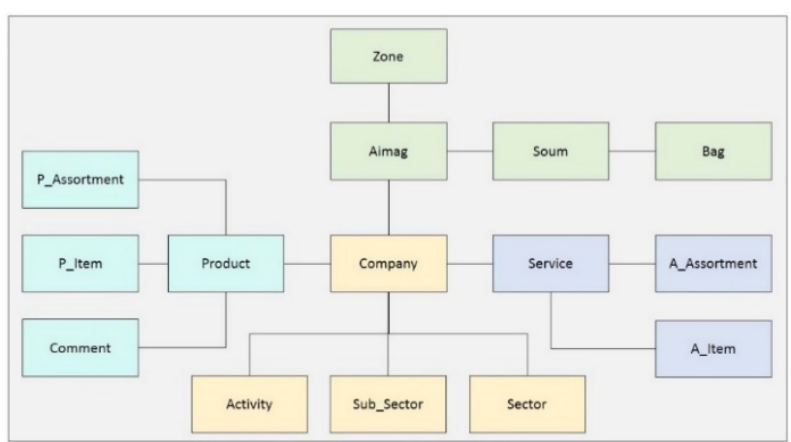

Figure 3. Result of the normalization

The physical model transfers the specified logical data to the internal data structure. At present, with the advancement of computer technology, UML method is being widely used for different database modelling. The UML is a standardized modeling language consisting of an integrated set of diagrams, developed to help system and software developers for specifying, visualizing, constructing, and documenting the artifacts of software systems as well as for other modeling purposes. It uses mostly graphical notations to express the design of software projects [7]. The most commonly used diagrams are class diagram, activity diagram, sequence diagram, and use case diagram.

In the present study, we have used a UML class diagram for physical modelling of the catalogue database. The class diagram is the most common diagram type that describes the structure of a system by showing the system's classes, their attributes, operations (if available) and the relationships among objects. For the catalogue database, the following have been thoroughly identified:

- $\quad$ Classes (i.e tables)

- $\quad$ Attributes (i.e. fields)

- Data types (i.e., integer, varchar, text, etc.)

- $\quad$ Relationships (1: m).

A final UML class diagram representing a physical model of the catalogue database is illustrated in Figure 4.

\section{SYSTEM DESIGN}

In any system design, at the first hand, a system boundary should be defined. In the case of the present study, the system boundary contains three main streams such as input, service-based system and output. Here, the host-admin should give the rights for data entry to the enterprises.

Based on the database contents and information analysis, we can elaborate a system structure. It should consist of the following 4 main parts:

1. System control: It will work on data and page creation. It will also manage admin module. All processes will be sent to Data Registration and Processing tab.

a. Data registration environment creator

b. Data processing

c. Administrator module

2. Data registration and processing: It will mainly work with database tools.

a. Registration module

i. Create content, edit and delete it

ii. Show contents, search and filter them

iii. Print and download

b. Report, analysis and dashboard module

i. Analysis

ii. Report 
c. Database contains necessary data sets

3. Content publisher and portal: It has the following parts:

a. Web portal
i. Menu
ii. Content
iii. Search, filter, etc.

b. Statistics and content publishing module

c. Mobile app

i. Menu

ii. Content iii. Search, filter, etc.

4. Additional module: It has the following parts:
a. System log
i. Input track
ii. Output track
iii. User $\log$
b. Notification module
c. Send notification
d. Notification action.

Usually, the IT experts and users are interested in system table design and its contents. Therefore, we decided to illustrate the table design of the system (Figure 5).

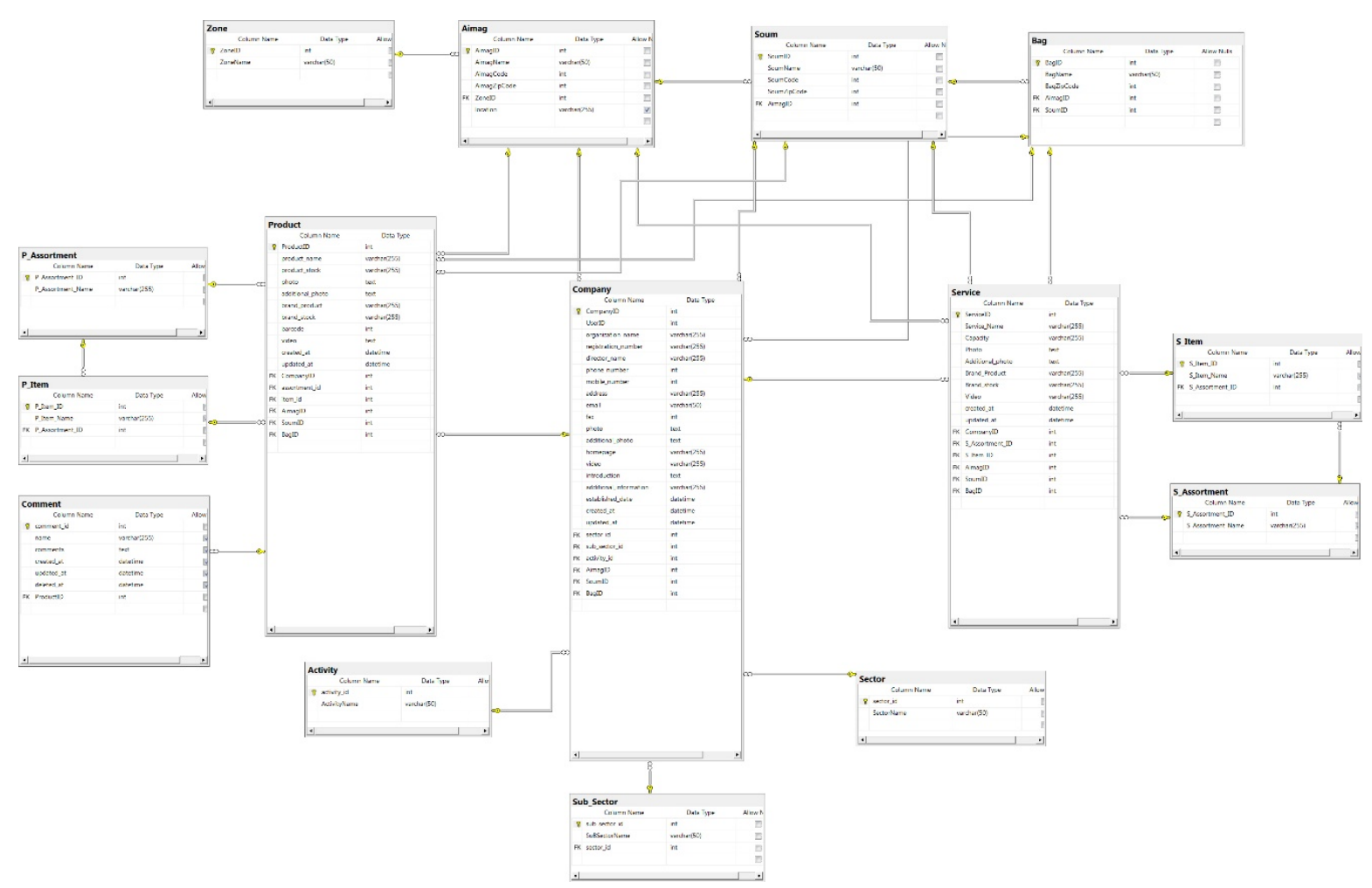

Figure 4. UML class diagram representing the physical 


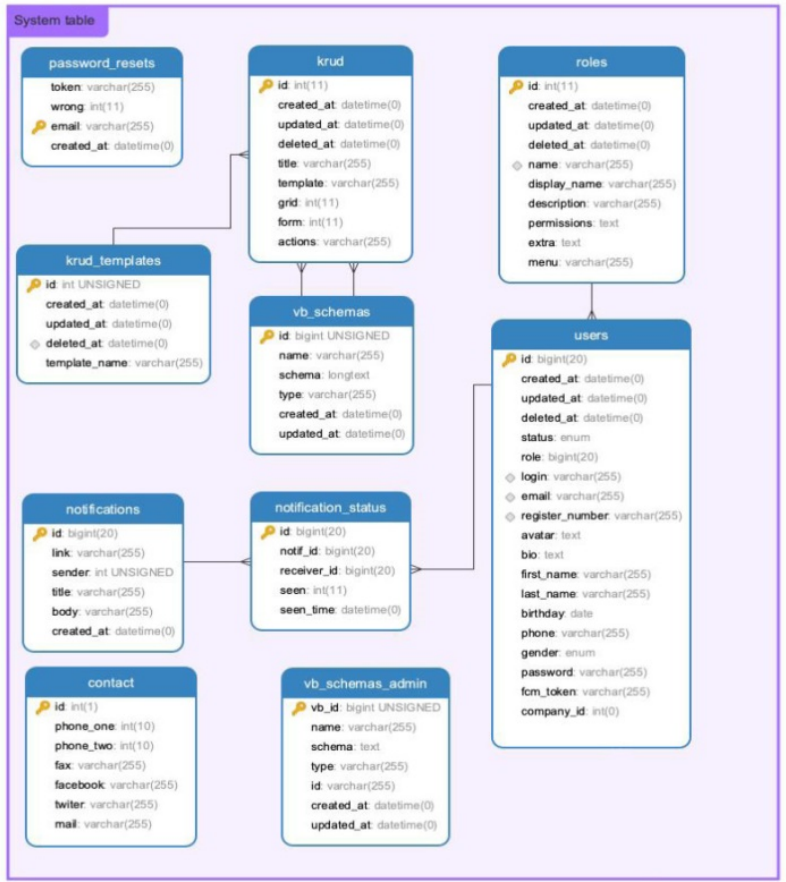

Figure 5. The system table design

\section{CONCLUSIONS}

At present, Mongolia has different needs to develop online service oriented geospatial information systems used for a variety of spatial related decision-making processes. In the country's context, the system could be implemented as a webbased dynamic software platform to enable the effective information sharing among various stakeholders and support national sector development operations. Database structures of these systems might be in different forms depending upon a number of requirements. Within the framework of the study, we described a conceptual framework and system design related to the service-based geospatial information system.

\section{REFERENCES}

[1] Amarsaikhan, D., Bolor, G., Dejidmaa, D., Enkhjargal, D. and Bayarbaatar, D., 2018, Open source-based and proprietary Web-GIS systems, Full paper published in Proceedings of the ACRS, Kuala Lumpur, Malaysia.

[2] Singh, S. and Singh, P., 2014, Modeling a geospatial database for managing travelers' demand, International Journal of Database Management Systems, Vol.6, No.2, pp.39-47. DOI: https://doi.org/10.5121/ijdms.2014.6203
[3] Amarsaikhan, D., 2014, Development of spatial data infrastructure of Mongolia, Final report of the project, pp. 102 .

[4] Project of ADB, 2020, Unleashing the private sector to drive inclusive growth in Eastern Mongolia. Final report of the ADB project.

[5] Bolorchuluun, 2015, GIS development and applications in Mongolia, Proceedings of the Third Conference "GIS-based Global History from Asian Perspectives", Sendai, Japan.

[6] Watt, A. and Eng.N., 2014, Database Design 2nd Edition. Victoria, B.C.: BCcampus. Retrieved from https:/opentextbc.ca/dbdesign01/.

[7] Amarsaikhan, D., Janzen, J., Egshiglen, E. and Gantuya, R., 2014, Urban land use change study in Mongolia using spatial techniques, International Journal of Sustainable Building Technology and Urban Development, Vol.5 (1), pp.35-43.

DOI: https://doi.org/10.1080/2093761X.2013.867823

[8] Lynch, W., 2020, A Comprehensive Guide to 14 Types of UML Diagram, Available: https://warren2lynch.medium.com/acomprehensive-guide-to-14-types-of-umldiagram-affcc688377e. 